\title{
Pregnancy Intention and Birth Outcomes: Does the Relationship Differ by Age or Race?
}

\author{
KRISTIE KEETON, M.D., M.P.H. ${ }^{1}$ and RODNEY A. HAYWARD, M.D. ${ }^{2}$
}

\begin{abstract}
Purpose: We hypothesized that intended pregnancy is associated with decreased risk of poor birth outcomes, irrespective of age or race.

Methods: We conducted a cross-sectional study of 47,956 live singleton births using 1993-2001 data from the Centers for Disease Control and Prevention's (CDC) Pregnancy Risk Assessment Monitoring System (PRAMS). We used logistic regression to estimate the odds of giving birth to a very low birth weight/very preterm infant (VLBW/VPT) or an infant admitted to the neonatal intensive care unit (NICU) by pregnancy intention.

Results: Intended pregnancy was associated with a slightly higher risk than unintended pregnancy of a VLBW/VPT birth in young white women ( $\leq 22$ years) and black women of all ages. Intended pregnancy was associated with a slightly higher risk of infant NICU admission in white women under age 28 but with a lower or equivalent risk of infant NICU admission for black women of all ages than unintended pregnancy. From ages 22 to 37, black women had a significantly higher risk of VLBW/VPT birth with intended pregnancies than white women, but from ages 18 to 32, white women were at significantly more risk than black women of requiring infant NICU admission with intended pregnancies.

Conclusions: Contrary to our hypothesis, an intended pregnancy at a young age was associated with a higher risk of poor birth outcomes.
\end{abstract}

\section{INTRODUCTION}

$\mathbf{I}_{\mathrm{t} w}^{\mathrm{N}}$ N THE UNITED STATES, black infants are more than twice as likely as white infants to die in the first year of life. Most of this disparity is related to black infants being about three times more likely to be very low birth weight $(<1500 \mathrm{~g})$ and very preterm $(<32$ weeks $) .{ }^{1}$ Although policymakers have attempted to reduce these differentials for decades, the gap remains wide, and the reasons for these differences are poorly understood.
One important factor to consider when investigating such differentials is pregnancy intention. An unintended pregnancy is one that is either mistimed (pregnancy occurred sooner than planned) or unwanted (pregnancy was not wanted at anytime). Unintended pregnancy is an important public health problem. Almost half of all pregnancies and approximately $22 \%$ of all births in the United States are unintended. ${ }^{2}$ The proportion of unintended childbearing varies among population subgroups. For example, wo-

\footnotetext{
${ }^{1}$ University of Michigan, F4835 Mott Hospital, Ann Arbor, Michigan.

${ }^{2}$ University of Michigan and VA Ann Arbor Healthcare System, Ann Arbor, Michigan.

K.K. conducted this research as a fellow in the Robert Wood Johnson Clinical Scholars Program at the University of Michigan with data from the Centers for Disease Control and Prevention's Pregnancy Risk Assessment Monitoring System.
} 
men with less than a high school population are over 3 times more likely to have an unintended birth than women with a college degree. Unintended childbearing is also high among adolescents of all races and among black women of all ages. $^{3}$

Research has linked unintended pregnancies with adverse maternal behavior during pregnancy, including late and insufficient prenatal care and smoking and drinking during pregnancy. ${ }^{4}$ Unintended pregnancy has also been associated with adverse perinatal outcomes, such as low birth weight and infant mortality. The consequences of unintended pregnancy may continue into childhood, and adverse health and social outcomes have been reported for both mother and child. ${ }^{4}$

As the rate of unintended pregnancy varies among population subgroups, so may the outcomes of these unintended pregnancies. Contrary to findings in other racial groups, older black adolescents have better birth outcomes on average than black mothers in their 20s and early 30s.., 6 One possible explanation for this is weathering, or early health deterioration among young black women. ${ }^{5}$ Another possible explanation for differences in birth outcomes may be differences in pregnancy intention. Does controlling for pregnancy intention in different population subgroups account for some of the variability in birth outcomes? We are unaware of research asking whether differences in pregnancy intention explain part of the differences in birth outcomes among population subgroups when categorized by both race and age.

We hypothesized that intending pregnancy at any age decreases the risk of poor birth outcomes. We were specifically interested in how pregnancy intention was associated with birth outcomes in different age groups (adolescents in particular) and in blacks compared with whites. Using data from the Center for Disease Control and Prevention's (CDC) Pregnancy Risk Assessment Monitoring System (PRAMS), we sought to characterize the relationship among intention, age, race, and birth outcomes.

\section{MATERIALS AND METHODS}

To assess the association of pregnancy intention, age, race, and birth outcomes, we conducted a cross-sectional study using data from the
PRAMS. In brief, PRAMS is a U.S. federal/state cooperative questionnaire of women who have had a recent live birth and is drawn from each state's birth certificate file. Each participating state samples between 1300 and 3400 women per year; some high-risk groups are oversampled. New mothers are sampled monthly from birth certificates and mailed a questionnaire 2-4 months after delivery. A second questionnaire is mailed to nonrespondents, and if nonresponse persists, attempts are made to administer the questionnaire, by telephone. The PRAMS database includes information from questionnaires and birth certificates. The PRAMS participating states, questionnaire and methods are described in detail elsewhere. ${ }^{7,8}$

Pregnancy intention was assessed by asking: Thinking back to just before you got pregnant, how did you feel about becoming pregnant? Unintended pregnancies were defined as pregnancies in which the respondent answered; I didn't want to be pregnant then or at any time in the future, or I wanted to be pregnant later. Intended pregnancies were defined as pregnancies in which the respondent answered: I wanted to be pregnant sooner, or I wanted to be pregnant then. Because of the relatively small numbers of pregnancies in adolescents, we were not able to use a multilevel intention variable (wanted, mistimed, unwanted); we dichotomized the intention variable, combining mistimed and unwanted pregnancies in our analysis. This dichotomy remains a valuable method of assessment because it allow for the analysis of differences among population subgroups. ${ }^{2}$

We used all data available from the CDC. This included any participating state from 1993 to 2001 that achieved an overall response rate and a response rate among adolescents of at least $70 \%$. States and years included were Alabama (1994-1996, 1998, and 2001), Florida (1993-2001), Georgia (1995-1996), Illinois (1998 and 20002001), Louisiana (1998), North Carolina (19971999), New Mexico (1998), Oklahoma (19951996), South Carolina (2000-2001), and Washington (1998-1999). A total of 58,473 births were included in the dataset.

Women who did not provide information on pregnancy intention $(n=3387)$ were excluded from our analyses. In addition, we excluded multiple pregnancies $(n=2926)$ and congenital anomalies $(n=1088)$, as both have a direct influence on our outcomes of interest. Because preg- 
nancy before 12 and after 44 years of age is rare, we excluded births to mothers in these age groups $(n=43)$. Because of the small number of respondents from other racial backgrounds, only black and white women were included in the study (excluding 3073 women from other racial backgrounds).

Our primary outcome variables were (1) a composite measure of very low birth weight (VLBW, birth weight of $<1500 \mathrm{~g}$ ) and very preterm birth (VPT, birth at $<32$ weeks completed gestational age) and (2) admission to the neonatal intensive care unit (NICU). We combined the VLBW and VPT variables because they were correlated $(r=$ 0.8 ). VLBW and VPT births were based on birth certificate data, and NICU admission was defined as answering yes to the PRAMS question: After your baby was born, was he or she put in the intensive care unit?

We used multivariable logistic regression to control for the following potential confounders: maternal age, marital status, tobacco use, alcohol use, receipt of prenatal care in the first trimester, total number of prenatal care visits, medical complications of pregnancy (hypertension or diabetes), and birth history (a combination of parity and history of prior preterm or low birth weight births). We did not include maternal education as a measure of socioeconomic status (SES) in our primary analyses, as it is an inaccurate measure of SES in adolescents. Alternatively, we used receipt of Medicaid (before pregnancy, during pregnancy, or both) and residential crowding (the ratio of the number of people living with the mother to the number of rooms in that residence) as a measures of SES. ${ }^{9}$ Women were classified as living in crowded conditions $(>1.0$ persons per room) or not living in crowded conditions $(\leq 1.0$ persons per room). ${ }^{10,11}$ We conducted subgroup analyses for women $>22$ years of age and included education as a potential confounder. We chose women $>22$ years for this analysis for two reasons: (1) to allow time for high school graduation and entry into or graduation from trade school or college and (2) because it is the integer midpoint of our third age category.

We calculated the relative risk increase (RRI) for intended pregnancy relative to unintended pregnancy by dividing the odds ratio for intended pregnancies $\left(\mathrm{OR}_{\mathrm{I}}\right)$ by the odds ratio for unintended pregnancies $\left(\mathrm{OR}_{\mathrm{U}}\right)\left(\mathrm{RR}_{\mathrm{I}}=\mathrm{OR}_{\mathrm{I}} / \mathrm{OR}_{\mathrm{U}}\right)$ at the integer midpoint of each age category. Age categories were determined a priori and driven by definitions of adolescent childbearing proposed by Phipps and Sowers. ${ }^{12}$ We used the integer of each age category (Table 1) and rounded up if necessary. Because the incidence of poor birth outcomes is low $(<5 \%)$, the ORs for these outcomes were close approximations of RRs. In addition to the confounders mentioned, the interaction effect of age and intention was included in the multivariable regression models.

We obtained an exemption from the Institutional Review Board of the University of Michigan before conducting analyses with a de-identified CDC dataset. STATA 8.0 was used to conduct all analyses. Weighted analysis was done to adjust for the complex survey design so that the results reported in this paper are representative of the population in the states studied.

\section{RESULTS}

The final PRAMS dataset analyzed included 47,956 live singleton births from 10 states between 1993 and 2001 that met our eligibility criteria.

Sociodemographic and medical characteristics, tabulated by intendedness and race, are presented in Table 1. The columns are in four categories: black women with intended pregnancies, black women with unintended pregnancies, white women with intended pregnancies, and white women with unintended pregnancies. The rows list characteristics of interest (e.g., age, Medicaid receipt, residential crowding). As shown in Table 1, $46.9 \%$ of pregnancies were among white women who intended pregnancy, followed by $29.4 \%$ of pregnancies among white women with unintended pregnancies; $16.3 \%$ of pregnancies were among black women with unintended pregnancies, and $7.4 \%$ of all pregnancies were among black women who intended pregnancy. In most categories, women with unintended pregnancies have a higher prevalence of risk factors for poor birth outcomes than women who intended their pregnancies.

Table 2 shows associations of an intended pregnancy with VLBW/VPT birth and NICU admission by race for all ages. After adjusting for potential confounding factors, intended pregnancy did not predict birth outcomes among white women. Intended pregnancy was, however, associated with a small increase risk of a VLBW/VPT birth but not NICU admission for black women compared with an unintended pregnancy. Re- 
Table 1. Estimated Characteristics of Mothers in Overall Population with Intended and Unintended Pregnancies, by Race, \% (95\% Confidence interval)

\begin{tabular}{|c|c|c|c|c|}
\hline & \multicolumn{2}{|c|}{ Black } & \multicolumn{2}{|c|}{ White } \\
\hline & $\begin{array}{c}\text { Intended } \\
(\mathrm{n}=5,061)\end{array}$ & $\begin{array}{l}\text { Unintended } \\
(\mathrm{n}=10,713)\end{array}$ & $\begin{array}{c}\text { Intended } \\
(\mathrm{n}=19,217)\end{array}$ & $\begin{array}{l}\text { Unintended } \\
(\mathrm{n}=12,821)\end{array}$ \\
\hline \multicolumn{5}{|l|}{ Maternal age, years } \\
\hline $12-15$ & $0.1(0.03-0.1)^{\mathrm{a}}$ & $0.5(0.5-0.6)$ & $0.1(0.1-0.1)$ & $0.4(0.3-0.4)$ \\
\hline $16-19$ & $0.7(0.6-0.8)$ & $4.4(4.2-4.6)$ & $2.6(2.5-2.8)$ & $5.4(5.2-5.7)$ \\
\hline $20-24$ & $1.9(1.8-2.1)$ & $5.5(5.3-5.7)$ & $9.4(9.0-9.8)$ & $9.1(8.7-9.4)$ \\
\hline $25-29$ & $2.0(1.9-2.1)$ & $3.2(3.0-3.4)$ & $14.4(14.0-14.9)$ & $7.1(6.8-7.5)$ \\
\hline $30-34$ & $1.7(1.6-1.9)$ & $1.7(1.6-1.8)$ & $13.4(12.9-13.8)$ & $4.9(4.6-5.1)$ \\
\hline $35-44$ & $0.9(0.8-1.1)$ & $1.0(0.9-1.2)$ & $7.0(6.7-7.4)$ & $2.5(2.3-2.7)$ \\
\hline Total & $7.4(6.6-8.0)$ & $16.3(15.4-17.2)$ & $46.9(45.1-48.8)$ & $29.4(27.8-30.9)$ \\
\hline \multicolumn{5}{|l|}{ Medicaid receipt } \\
\hline Yes & $3.9(3.7-4.1)$ & $12.1(11.8-12.3)$ & $12.2(11.8-12.7)$ & $15.3(14.9-15.7)$ \\
\hline No & $3.5(3.3-3.6)$ & $4.2(4.0-4.4)$ & $34.7(34.3-35.1)$ & $14.1(13.7-14.5)$ \\
\hline \multicolumn{5}{|l|}{ Residential crowding } \\
\hline$\leq 1$ persons per room & $6.0(5.8-6.1)$ & $12.4(12.1-12.6)$ & $43.7(43.4-44.0)$ & $25.7(25.3-26.0)$ \\
\hline$>1$ persons per room & $1.2(1.0-1.3)$ & $3.7(3.5-3.9)$ & $3.6(3.3-3.9)$ & $3.9(3.7-4.2)$ \\
\hline \multicolumn{5}{|l|}{ Marital status } \\
\hline Married & $3.8(3.6-4.0)$ & $3.7(3.5-3.9)$ & $40.2(39.8-40.5)$ & $17.3(17.0-17.7)$ \\
\hline Single & $3.6(3.4-3.7)$ & $12.7(12.4-12.9)$ & $6.8(6.4-7.1)$ & $12.0(11.6-12.4)$ \\
\hline \multicolumn{5}{|l|}{ Tobacco use $\mathrm{b}^{\mathrm{b}}$} \\
\hline Yes & $0.4(0.3-0.5)$ & $1.0(0.9-1.1)$ & $4.5(4.2-4.8)$ & $5.2(4.9-5.5)$ \\
\hline No & $7.0(6.8-7.2)$ & $15.4(15.2-15.7)$ & $42.4(42.1-42.8)$ & $24.1(23.8-24.4)$ \\
\hline \multicolumn{5}{|l|}{ Alcohol use ${ }^{b}$} \\
\hline Yes & 0 & $0.10 .1-0.1)$ & $0.2(0.2-0.3)$ & $0.1(0.1-0.2)$ \\
\hline No & $7.4(7.2-7.5)$ & $16.3(16.1-16.6)$ & $46.7(46.4-46.9)$ & $29.1(28.9-29.4)$ \\
\hline \multicolumn{5}{|l|}{ First trimester $\mathrm{PNC}^{\mathrm{c}}$} \\
\hline Yes & $5.6(5.4-5.8)$ & $9.1(8.9-9.4)$ & $40.7(40.4-41.1)$ & $20.2(19.8-20.6)$ \\
\hline No & $1.7(1.6-1.8)$ & $7.1(6.8-7.3)$ & $6.3(6.0-6.6)$ & $9.2(8.9-9.6)$ \\
\hline \multicolumn{5}{|l|}{ PNC visits } \\
\hline 0 & $0.1(0.1-0.2)$ & $0.5(0.4-0.7)$ & $0.7(0.5-0.9)$ & $0.6(0.4-0.8)$ \\
\hline $1-8$ & $0.6(0.5-0.7)$ & $1.9(1.7-2.1)$ & $2.5(2.2-2.8)$ & $2.5(2.2-2.8)$ \\
\hline $9-20$ & $5.1(4.8-5.3)$ & 10.7 (10.4-11.0) & $40.8(40.2-41.4)$ & $23.1(22.6-23.6)$ \\
\hline$>20$ & $1.1(0.9-1.2)$ & $2.4(2.2-2.6)$ & $4.5(4.1-4.9)$ & $2.9(2.5-3.2)$ \\
\hline \multicolumn{5}{|l|}{ Medical risk factors ${ }^{\mathrm{d}}$} \\
\hline Present & $0.6(0.5-0.6)$ & $0.9(0.8-0.9)$ & $2.8(2.6-3.0)$ & $1.6(1.4-1.7)$ \\
\hline Absent & $6.8(6.6-7.0)$ & $15.5(15.2-15.7)$ & $44.2(43.9-44.5)$ & $27.8927 .5-28.1)$ \\
\hline \multicolumn{5}{|l|}{ Birth history } \\
\hline First & $3.0(2.9-3.2)$ & $6.6(6.4-6.8)$ & $21.1(20.6-21.6)$ & $12.9(12.5-13.3)$ \\
\hline Previous normal & $3.3(3.1-3.5)$ & $7.2(7.0-7.5)$ & $22.5(22.0-23.0)$ & $14.2(13.8-14.6)$ \\
\hline Previous LBW or PT & $1.0(0.9-1.1)$ & $2.4(2.3-2.6)$ & $3.4(3.2-3.7)$ & $2.3(2.1-2.5)$ \\
\hline
\end{tabular}

a\% (95\% confidence interval).

${ }^{\mathrm{b}}$ At any time during pregnancy.

CPNC, prenatal care.

dDiabetes, hypertension, or other medical risk factors during pregnancy.

sults did not differ when level of maternal education was included in alternative analysis limited to women $>22$ years of age (data not shown).

Despite the lack of an average overall effect among white women, the association between pregnancy intention and birth outcomes varies considerably across age (Table 3). White adolescents who intended their pregnancy had an increased risk of VLBW/VPT births compared with black adolescents who intended pregnancy. This association de- creases with advancing age. In contrast, the risk of VLBW/VPT births for black women who intended pregnancy is remarkably similar across age. At age 18 , white women have a significantly higher risk of giving birth to a VLBW/VPT infant than do black women who intend pregnancy. This trend reverses at age 22; thereafter, among women who intend pregnancy, black women have a significantly higher risk of giving birth to a VLBW/VPT infant than do white women of the same age. 
Table 2. Relative Risk (95\% CI) OF VLBW/VPT BiRTH and NICU Admission Associated with Pregnancy InTENTiOn by Maternal Race, Ages $12-44^{\text {a }}$

\begin{tabular}{lcc}
\hline & Black & White \\
\hline VLBW/VPT & $1.19(1.02-1.38)$ & $1.08(0.92-1.30)$ \\
NICU admission & $0.93(0.81-1.07)$ & $1.08(0.96-1.21)$ \\
\hline
\end{tabular}

aAdjusted for maternal age, Medicaid receipt, residential crowding, marital status, tobacco and alcohol use, birth history, medical risk factors during pregnancy, and receipt of prenatal care.

Intending pregnancy seems to have a slightly protective effect against NICU admission with advancing age. Although the general trend of decreasing risk by advancing age is similar for black and white women, the magnitude of the risk differs. Among women with intended pregnancy, white women aged 18-32 are at significantly higher risk for NICU admission than black women of the same age.

\section{DISCUSSION}

We sought to determine if pregnancy intention explained age or racial differences in birth outcomes. Contrary to our a priori hypothesis, intending pregnancy was not always associated with better birth outcomes. In fact, intending pregnancy was associated with increased risk of VLBW/VPT births and NICU admission in white adolescents and blacks of all ages. Our results differ from prior published results, which found increased risks of adverse maternal behaviors ${ }^{13}$ and poor pregnancy outcomes with unintended preg- nancy. ${ }^{14-19}$ We are the first to report findings in subgroup analysis, and this may be one explanation for the discrepancy between our results and the results of others.

Other possible reasons for these findings are as follows. First, the meaning of intention might differ by age and race. Because of differing social meanings of pregnancy by age, the intention to become pregnant in adolescence may be either a marker of social risk or a marker of social support, neither of which was fully captured in our models. For example, pregnancy among black adolescents is more common relative to pregnancy among white adolescents. A black adolescent who intends pregnancy may have more social support, that is, availability of family members (mothers, grandmothers, aunts), to help raise their children. Alternatively, an intended pregnancy in a white adolescent may be a stronger marker for overall social dysfunctionality, identifying individuals with higher rates of other risky behaviors, such as heavier drug or alcohol abuse. A second possibility is that by controlling for behavioral variables (tobacco and alcohol use, receipt of prenatal care), we may have accounted for important intermediaries between unintended pregnancies and poor birth outcomes. Also, pregnancies that end in abortion are not captured in this dataset. Women who decide to continue unintended pregnancies may be different in ways that provide less risk of a poor outcome than women who seek termination of unintended pregnancies. In other words, the availability of abortion mitigates the risk of adverse birth outcomes due to unintended pregnancies by providing an alternative for those at the highest risk.

Table 3. Relative Risk Increase (RRI ${ }^{a}$ ) of VLBW/VPT Birth and NiCU Admission for Intended Relative to Unintended Pregnancies at (Integer) Midpoint of Each Age Category, by Race ${ }^{b}$

\begin{tabular}{|c|c|c|c|c|c|c|}
\hline \multirow[b]{2}{*}{ Age, years } & \multicolumn{3}{|c|}{$V L B W / V P T$} & \multicolumn{3}{|c|}{ NICU admission } \\
\hline & Black & White & $\mathrm{p}$ value & Black & White & $\mathrm{p}$ value \\
\hline 14 & 1.18 & 1.27 & 0.26 & 1.00 & 1.34 & 0.98 \\
\hline 18 & 1.18 & 1.20 & $<0.01^{*}$ & 0.98 & 1.24 & $<0.01^{*}$ \\
\hline 22 & 1.18 & 1.14 & $<0.01^{*}$ & 0.96 & 1.15 & $<0.01^{*}$ \\
\hline 27 & 1.19 & 1.-07 & $<0.01^{*}$ & 0.93 & 1.06 & $0.03^{*}$ \\
\hline 32 & 1.19 & 1.00 & $<0.01^{*}$ & 0.91 & 0.97 & $<0.01^{*}$ \\
\hline 37 & 1.19 & 0.93 & $<0.01^{*}$ & 0.89 & 0.88 & $<0.01^{*}$ \\
\hline
\end{tabular}

aRRI, OR for intended pregnancies/OR for unintended pregnancies $\mathrm{OR}_{\mathrm{I}} / \mathrm{OR}_{\mathrm{U}}$.

bAdjusted for maternal age, Medicaid receipt, residential crowding, marital status, tobacco and alsohol use, birth history, medical risk factors during pregnancy, and receipt of prenatal care.

*Relative risk increase between black and white is significantly different at $p<0.05$. 
This study has several limitations. First, the study is an observational study, and unmeasured or unidentified confounding factors could affect associations. Second, the PRAMS dataset does not include information on unintended pregnancies that ended in abortion, miscarriage, or stillbirth. Conclusions cannot be drawn about or applied to women whose pregnancy did not result in a live birth. Third, several issues could potentially bias a retrospective measure of pregnancy intention. Recall bias is a major concern, as respondents are asked to report events and feelings that may have occurred up to 15 months previously. Reports of intention are likely to be influenced by the presence of the infant; thus, intendedness may be difficult to quantify, resulting in poor reliability. ${ }^{20}$ Feelings of intention are also subject to other external influences (i.e., fear of losing an already sick child, feelings of the partner or father of the baby), ${ }^{21}$ and, thus, intention is likely to change during and after pregnancy. ${ }^{22,23}$ Futhermore, birth outcomes may vary by the severity of mistiming (moderately mistimed, seriously mistimed, and unwanted). ${ }^{19}$ Fourth, respondents may alter responses to sensitive topics.

Our results suggest, however, that white adolescents who report intending pregnancy have poorer birth outcomes than those who do not. Uncovering the causal pathways underlying this association has the potential to improve our understanding of preventable causes of poor birth outcomes.

\section{ACKNOWLEDGMENTS}

We thank Joel Howell and Rudi Ansbacher for critical review and editorial assistance, Sonya DeMonner for technical assistance, and the PRAMS Working Group and the CDC for access to data and assistance with the analyses.

\section{REFERENCES}

1. March of Dimes. Perinatal health in the United States. peristats.modimes.org/ 2003.

2. Finer LB, Henshaw SK. Disparities in rates of unintended Pregnancy in the United States, 1994 and 2001. Perspect Sex Reprod Health 2006;38:90.

3. Chandra A, Martinez GM, Mosher WD, Abma JC, Jones J. Fertility, family planning, and reproductive health of U.S. women: Data from the 2002 National Survey of Family Growth. Vital \& Health Statistics,
Series 23, Data from the National Survey of Family Growth, 2005:1.

4. Brown SS, Eisenberg L. IOM. The best intentions: Unintended pregnancy and the well-being of children and families. Washington, DC: National Academy Press, 1995.

5. Geronimus AT. Black/white differences in the relationship of maternal age to birthweight: A population-based test of the weathering hypothesis. $\underline{\text { Soc Sci }}$ Med 1996;42:589.

6. Rauh VA, Andrews HF, Garfinkel RS. The contribution of maternal age to racial disparities in birthweight: A multilevel perspective. Am J Public Health 2001;91:1815.

7. CDC. Pregnancy risk assessment monitoring system. www.cdc.gov/reproductivehealth/srv_prams.htm 2003

8. Gilbert BC, Shulman HB, Fischer LA, Rogers MM. The Pregnancy Risk Assessment Monitoring System (PRAMS): Methods and 1996 response rates from 11 states. Matern Child Health J 1999;3:199.

9. Gazmararian JA, Adams MM, Pamuk ER. Associations between measures of socioeconomic status and maternal health behavior. Am J Prev Med 1996:108.

10. Centerwall BS. Race, socioeconomic status, and domestic homicide, Atlanta, 1971-72. Am J Public Health 1984;74:813.

11. Characteristics of housing units. Washington, DC: Department of Commerce, 1982.

12. Phipps MG, Sowers M. Defining early adolescent childbearing. Am J Public Health 2002;92:125.

13. Weller RH, Eberstein IW, Bailey M. Pregnancy wantedness and maternal behavior during pregnancy. Demography 1987;24:407.

14. Williams LB. Determinants of unintended childbearing among ever-married women in the United States: 1973-1988. Fam Plann Perspect 1991;23:212.

15. Morris NJ, Udry R, Chase H. Reduction of low birth weight rates by prevention of unwanted pregnancies. Am J Public Health 1973;63:934.

16. Laukaran VH, van den Berg BJ. The relationship of maternal attitude to pregnancy outcomes and obstetric complications. A cohort study of unwanted pregnancy. Am J Obstet Gynecol 1980;136:374.

17. Marsiglio W. Does wanting to become pregnant with a first child affect subsequent maternal behaviors and infant birth weight? J Marriage Fam 1988;50:1023.

18. Korenman S, Kaestner R, Joyce T. Consequences for infants of parental disagreement in pregnancy intention. Perspect Sex Reprod Health 2002;34:198.

19. Pulley L, Klerman LV, Tang H, Baker BA. The extent of pregnancy mistiming and its association with maternal characteristics and behaviors and pregnancy outcomes. Perspect Sex Reprod Health 2002;3 4:206.

20. Sable MR. Pregnancy intentions may not be a useful measure for research on maternal and child health outcomes. Family 1999;5:249.

21. Williams LB. Determinants of couple agreement in U.S. fertility decisions. Fam Plann Perspect 1994; 26:169. 
22. Santelli J, Rochat $R$, Hatfield-Timajchy $K$, et al. The measurement and meaning of unintended pregnancy. Perspect Sex Reprod Health 2003;35:94.

23. Poole VL, Flowers JS, Goldenberg RL, Cliver SP, McNeal S. Changes in intendedness during pregnancy in a high-risk multiparous population. Matern Child Health J 2000;4:179.
Address reprint requests to: Kristie Keeton, M.D., M.P.H. University of Michigan F4835 Mott Hospital Ann Arbor, MI 48109-0264 E-mail: kristie@umich.edu 
This article has been cited by:

1. Jessica D. Gipson, Michael A. Koenig, Michelle J. Hindin. 2008. The Effects of Unintended Pregnancy on Infant, Child, and Parental Health: A Review of the Literature. Studies in Family Planning 39:1, 18-38. [CrossRef] 